\title{
Carnets
}

Revue électronique d'études françaises de l'APEF

Première Série - $1 \mid 2009$

La mer... dans tous ses états

\section{Du limen marin dans l'écriture de Jean Lods}

\section{Annick Gendre}

\section{(2) OpenEdition}

\section{Journals}

Édition électronique

URL : http://journals.openedition.org/carnets/2918

DOI : 10.4000/carnets.2918

ISSN : 1646-7698

Éditeur

APEF

Édition imprimée

Pagination : 91-116

Référence électronique

Annick Gendre, « Du limen marin dans l'écriture de Jean Lods », Carnets [En ligne], Première Série -

1 | 2009, mis en ligne le 13 juin 2018, consulté le 01 mai 2019. URL : http://journals.openedition.org/ carnets/2918; DOI : 10.4000/carnets.2918

\section{(c) (i) (8)}

Carnets est mis à disposition selon les termes de la licence Creative Commons - Atribution - Pas d'utilisation commerciale 4.0 International. 


\title{
DU LIMEN MARIN DANS L’ECRITURE DE JEAN LODS
}

\author{
ANNICK GENDRE \\ Université Michel de Montaigne - Bordeaux III \\ gendre.annick@free.fr
}

\begin{abstract}
Résumé
Composés entre 1973 et 1994, les récits de Jean Lods mettent en œuvre une représentation aussi riche que complexe de l'élément marin. La mer imaginaire qu'ils dessinent est le fait d'une subtile combinaison de la mer du Nord et de l'océan Indien. Diamétralement opposées géographiquement, ces deux immensités liquides ne donnent pas lieu à une dialectique nord-sud binaire, mais jouent un rôle majeur dans la partition de l'espace fictionnel. De toutes les ambivalences marines, nous avons retenu celle qui associe l'avalage et le pouvoir génésique. Si elle met cette partition en action, elle permet de mieux saisir l'enjeu de la représentation de la mer non seulement dans les histoires relatées, mais également dans le tissu du texte et dans l'écriture de l'auteur.
\end{abstract}

\section{Abstract}

Published between 1973 and 1994, the novels of Jean Lods elaborate a rich, complex representation of the sea element. The imaginary sea they depict is a subtle combination of the North Sea and the Indian Ocean. Opposed geographically, these two liquid immensities do not generate a binary North/South dialectics, but they have a significant role in the partition and in the score of the fictional space. Among all of the ambivalences related to the sea, we focused on the one combining orality and the genesic power, which both triggers off this partition, sometimes this score, and allows us to understand better the significance of the representation of the sea not only in the stories, but also in the author's writing.

Mots-clés: Limen marin, Mer du Nord, Océan Indien, Spatialité, Référentialité textuelle, Espace littéraire

Keywords: Sea limen, The North Sea, Indian Ocean, Spatiality, Textual referentiality, Literary space 
Et je suis le vent et le ciel...

Je suis le bateau et la mer...

Pourtant ce n'est pas moi...

Et je veux l'ignorer.

Fernando Pessoa

Midi. En bas la mer, étincelante et sombre à force de lumière. Gouffre patient.

Lorand Gaspar

Les récits de Jean Lods, publiés entre 1973 et 1994, livrent une représentation de l'élément marin déterminée par l'insularité qui présente la singularité de se construire à la faveur de la superposition de deux îles dont la localisation est diamétralement opposée: l'île de La Réunion et une île de la mer du Nord ${ }^{1}$. La représentation de celle-ci doit beaucoup à l'île de Texel dans laquelle réside l'auteur plusieurs mois par an depuis 1980.

L'île de fiction constitue une composante de l'élément qui la délimite, tout en constituant une promesse d'ailleurs, d'ouverture sur le reste du monde, la fin de l'isolement, si bien que les fictions n'ont de cesse de l'investir en tant que limen. En raison de sa polysémie, ce terme est probablement le plus à même de rendre compte de la complexité des représentations de l'élément dans ces récits. II désigne en effet à la fois le commencement et la fin, le dedans et le dehors (l'entrée et la maison), ce qui ouvre un passage et ce qui lui fait obstacle (la porte et la barrière). La terra insula est incognita grâce à l'immensité liquide qui l'indivise. Cette liquidité seuil donne lieu à une appropriation particulière de l'espace-terre. L'implantation dans l'île, nordique ou indiaocéanique, semble perçue inconsciemment comme une pénétration symbolique. Ce schème est intimement lié à la dialectique de la mer et de la terre: la mer efface ou fait apparaître, quand la terre repousse, rejette et quelquefois, abrite. Ajoutons à ces remarques liminaires la richesse des marines lodsiennes: phare et digues, galets et dunes, mer létale ou démontée, tous ces composants appellent une palette de luminosités aussi nuancées que variées, de même que leur gamme infinie de sonorités se décline au gré des événements de l'histoire, sans que soit oublié le silence qu'elles menacent.

Ce que nous désignons par l'expression "mer lodsienne" résulte d'une subtile combinaison de la mer du Nord et de l'océan Indien. Bien distincts, l'une et l'autre tantôt s'opposent, tantôt se complètent, tantôt se superposent. Dans la représentation imaginaire,

\footnotetext{
${ }^{1}$ Les analyses soumises ici sont le fruit de travaux de recherches réalisés dans le cadre de notre thèse de doctorat. Elles mettent en relief et prolongent les perspectives dégagées relatives à l'élément marin dont nous avons étudié le rôle dans la complexité du système dialectique de la spatialité lodsienne. Cf. Gendre, Annick, La Représentation de soi à travers la textualisation de l'espace insulaire réunionnais: étude de l'œuvre de Jean Lods, 2007, 812 p., Thèse de doctorat [en ligne], in: TEL [consulté le 17 décembre 2008] <URL:http://tel.archivesouvertes.fr/index.php?halsid=56k1cirvvrtr10qsrofcqtb0I7\&view_this_doc=tel-00174119\&version=1>.
} 
les deux mers tendent à n'en faire qu'une. Sans doute est-ce pour cela que la mer indiaocéanique brise avec la stéréotypie exotique véhiculée par le commerce touristique. Pour ce qui est de leur littérarité, l'une comme l'autre ne sont pas non plus dotées des clichés qui consistent à personnifier l'élément, comme dans les vers d'Auguste Lacaussade ou dans les strophes de Guy Agénor². L'appréhension subjective qu'en ont les personnages diffère de celle que se plaît à explorer Leconte de Lisle (cf. "Le Chant du nègre pêcheur", par exemple). Cette double mer, nordique et indiaocéanique, offre des tableaux fort différents des marines romantiques. Rarement létale, elle augure volontiers d'un mouvement. Force est de constater qu'elle joue un rôle déterminant dans le climat de certaines scènes, cependant que sa présence est évoquée de façon récurrente, en dehors de ces mêmes scènes romanesques. Lorsqu'elle est porteuse de l'ailleurs, de l'autre, la façon dont elle est connotée dépend de l'appréhension de l'altérité (étrangeté, point de fuite, ...). Entre-deux espace inquiétant, l'élément marin n'est pas considéré comme un écrin qui renfermerait des aires d'asile.

Le point de vue qui observe et vit l'élément marin dans cette écriture inscrit la mer dans une géographie imaginaire au sein de laquelle l'île du Nord et l'île tropicale s'explorent l'une et l'autre, l'une dans l'autre. Cette partition marine se joue des nombreuses ambivalences qui caractérisent l'élément. C'est la dialectique de l'avalage ${ }^{3}$, ou incorporation orale, et de la fonction génésique attribuée à l'élément marin qui retient ici notre attention, pour ses incidences sur la géographique imaginaire, dans la diégèse et dans le tissu du texte. L'espace-seuil de la mer y est orchestré de telle façon qu'il constitue un véritable actant. Enfin, l'étude de la référentialité textuelle de la surface marine permet de dégager l'enjeu fondamental de cette représentation. II importe de déplier grâce à ces trois étapes comment l'élément, saisi en tant qu'espace, s'impose comme limen. Comment la mer contribue-t-elle dans ces récits à la construction d'un espace-texte et comment participe-telle de l'espace littéraire ${ }^{4}$ ?

\section{La partition marine}

Le partage géographique auquel participe la mer lodsienne ne s'inscrit pas dans un clivage binaire nord-sud. II en va d'une autre partition de l'espace: comme en mathématiques, si la mer du Nord et l'océan Indien sont disjoints, ou plutôt distincts, leur

\footnotetext{
${ }^{2}$ Cités respectivement par Prosper Eve (2003: 30-32).

3 Terme couramment employé en psychanalyse, en anthropologie et en sociologie, cf. par exemple Gilbert Durand (1990: 233-237).

${ }^{4}$ Ces lignes de Maurice Blanchot peuvent être retenues pour définir ce que nous désignons par cette expression: "Et l'apparition dit précisément que quand tout a disparu, il y a encore quelque chose: lorsque tout manque, le manque fait apparaître l'essence de l'être qui est d'être encore là où il manque, d'être en tant que dissimulé..." (Blanchot, 1998: 340).
} 
réunion dans la spatialité imaginaire reconstitue celle-ci. La spatialité désigne ici l'ensemble des traits et des paramètres qui se réfèrent à l'espace. On ne peut se demander ce que crée le lieu de la mer, sans tenir compte du lieu depuis lequel il est perçu. Or, dans les récits qui nous intéressent, la représentation de la mer cultive la menace qui pèse sur les lisères permettant de l'identifier. Le Bleu des vitraux et Sven sont particulièrement éloquents à cet effet. Dans le premier de ces deux récits, Yann Toulec, le narrateur-personnage, convoque, à l'occasion de l'enterrement de sa mère, Anne-Sylvie, les tragédies qui ont marqué l'enfance de "Yannou". Ce dédoublement fécond que permettent les réminiscences offre une mise en scène la famille Toulec qui s'est enrichie grâce à l'esclavage. La fin de l'enfance coïncide avec la fin du régime colonial. Dans cette intrigue familiale, le fils s'efforce de comprendre ce qui a motivé le suicide de son père, René Toulec. Sven relate une habile mise en perspective de trois pères prisonniers de l'attente désœuvrée du retour de leurs enfants respectifs. Dans l'île nordique, le gardien du phare attend sa fille, Anne; l'ornithologue, Emmanuel, prépare l'arrivée de son fils, Sven, qui réside à La Réunion dans la vieille demeure familiale avec son propre père vieillissant, qui espère quant à lui le retour d’Emmanuel. Le récit décline ainsi une nouvelle boucle de l'attente paternelle.

La mer lodsienne est toujours envisagée d'un point de vue situé en surplomb, audessus de l'eau. L'observation implique que le corps du sujet regardant se situe en hauteur, tandis que son regard suit une trajectoire descendante. Cette double posture crée une distanciation à l'égard de l'objet observé. Elle semble parallèlement indiquer une sorte de hiérarchie spatiale: l'aire des humains, en hauteur, s'oppose au contrebas que la mer et l'océan investissent. A La Réunion, les infrastructures routières, comme le moindre chemin, tissent leur réseau vers les hauts: père et fils, dans Le Bleu des vitraux, "cheminent sur l'étroit ruban de pierre qui domine la mer" (Lods, 1887: 125). La grande maison domaniale surplombe également l'océan. Le choix de cet emplacement opéré par les grands propriétaires a souvent été interprété comme le fruit d'une représentation géographique de la pyramide sociale. Mais de telles analyses semblent à la fois hâtives et datées. Elles ne tiennent pas compte des rigueurs climatiques et des avaries tropicales. De plus, les romanciers peuvent avoir à cœur d'accorder une dimension symbolique au site de la grande maison coloniale. Dans Quelques Jours à Lyon, la "maison de la famille" du père du personnage principal se trouve, elle aussi, "sur la colline dominant l'océan" (Lods, 1994a: 156). Elle offre un écho aux nombreuses maisons domaniales des récits antérieurs de Jean Lods, et notamment à celle des Toulec, qui est située au cœur de la toile de leur puissance ${ }^{5}$ (Lods, 1987). Ce récit est le seul qui a été écrit exclusivement à la troisième personne. De ce fait, à la différence des autres textes de l'auteur, il supporte mieux d'être classé dans la

\footnotetext{
${ }^{5}$ Cf. l'étude de la grande maison coloniale, in Gendre, 2007: 475-492.
} 
catégorie générique du "roman". Le territoire de l'enfance y est double: Lyon où Romain Durieux a effectivement résidé avec sa mère jusqu'à sa majorité, et l'île de La Réunion, terre rêvée et fantasmée où vivait son père, célèbre écrivain régional dont les textes se sont distingués pour leur réalisme exotique.

Ainsi la maison surplombe-t-elle, mais pour mieux constituer un bastion dans le cirque. L'ornithologue de l'archipel des Wadden reproduit le même clivage vertical dans la représentation qu'il propose de l'île nordique, plus sauvage, lorsqu'il relate ses déplacements: "Je quittai l'enclos et marchai en direction du plateau qui dominait l'océan" (Lods, 1991: 219). Ce clivage explique probablement pourquoi l'élément marin revêt davantage les aspects d'une direction, au lieu d'être délimité comme un espace à part entière. Deux évocations des déplacements de la voiture de René Toulec, l'unique héritier, expriment le même éloignement du rivage: "Il abandonne le littoral" (Lods, 1987: 46) et "Il traverse Sainte-Suzanne, il traverse Saint-André, il abandonne le littoral et s'enfonce dans les montagnes" (ibid.: 161). La mer et la chaîne du cirque sont contiguës. Le même verbe "abandonner" est employé pour exprimer à la fois la distance prise à l'égard de la rive et le retrait dans les terres. Synonyme de renoncer à un bien, à un droit, à une hypothèse, le terme abandonner suppose un choix, une décision du sujet qui réalise l'action. Le littoral, en tant que direction, appelle une décision de renoncement. On ne renonce qu'à ce que l'on a accepté et convoité dans un premier temps. L'abandon volontaire de la direction du littoral coïncide, dans la vie de René, avec la tentative de renoncer à un désir. Au début du récit, le jeune homme surpris par l'amour "hors norme" qu'il éprouve pour Anne-Sylvie, se hâte de trouver asile dans la maison de son enfance, blottie dans le cirque (ibid.: 47). Dans cette retraite, il recouvre la force d'investir sa passion pour cette fille $d u$ feu, originaire de la France continentale, qui vit seule et qui travaille pour subvenir à ses besoins. René renonce aux critères bienséants de son milieu socio-familial. Mais peu avant son suicide, c'est pour se protéger cette fois de l'abandon d'Anne-Sylvie qui rentre seule définitivement pour la métropole, que René entraîne son fils dans sa fuite vers le cirque (ibid.: 161) et qu'il effectue dix ans plus tard le même trajet. Le littoral est, dans cette dernière phrase, l'espace qui manifeste l'abandon, celui d'où l'on aperçoit le paquebot qui s'éloigne.

De même, dans Sven, les dunes septentrionales constituent un site en surplomb grâce aux angles que présentent leurs sommets. Leur ligne évoquée de façon récurrente constitue un repère dans la continuité de l'espace insulaire du nord. Emmanuel se plaît à suivre son tracé: "Je me mis à marcher sur la crête des dunes" (ibid:: 85) et "ll marche, courbé à cause du vent, sur la crête de dunes" (ibid:: 170). La hauteur de sable qu'elle représente ne tarde pas à devenir un site stratégique. Tout à fait emblématique, elle est le lieu où s'opère la mise en abîme, la rencontre des fantaisies des personnages et les fantaisies relatives à leurs rencontres. La déhiscence de ce point appartient aussi aux 
retrouvailles du père et de son fils, ou de son double enfant. L'espace lodsien n'est pas un décor, il fait partie intégrante des événements dont il est à la fois le témoin et l'empreinte. Ici, grâce à la poésie et à la fantaisie, l'événement qui n'adviendra pas a déjà eu lieu.

La représentation de l'île de la mer du Nord s'enrichit de la partition verticale que réalise une connivence singulière entre la mer et le ciel. Baudelaire ne renierait pas le ciel de l'île, même si au lieu de peser sur un décor urbain, il adoucit ici l'ocre des dunes. A l'instar des oiseaux, l'humidité dont il est gorgé semble ne faire qu'escale sur l'île. Comme l'auteur, Emmanuel, le narrateur-personnage de Sven, a passé son enfance dans une île tropicale. Probablement est-ce pour cette raison qu'il se montre particulièrement sensible à la soudaineté des averses: "Dehors, l'énorme nuage noir, qui depuis un moment se déployait entre l'horizon et l'île, creva d'un coup" (Lods, 1991: 11). Dès la première page, cet indice fait le lien entre les deux îles et permet d'apprécier la qualité du regard que pose l'ornithologue sur l'île nordique: il la saisit dans ce qu'elle présente de similaire à l'île "verticale". Construit en fonction de cette île de l'enfance, son regard ne cesse d'impliquer implicitement ou explicitement une comparaison entre les deux espaces insulaires. Ces averses brutales et récurrentes sur l'île contribuent à la distinguer du continent: "Le grain avait passé l'île et ses nuages plombaient le continent" (ibid.: 14). Elles différencient ces deux qualités de terres, l'une ancrée dans le sol et sûre de ses prolongements, l'autre toujours sur le point de disparaître.

A l'instar de l'étendue marine, l'île nordique ne retient pas les météores. Sans ses résidants pour témoins, les phénomènes qui s'y produisent demeureraient dans l'ignorance de tous. Tout ce qui s'y passe dépend de l'attention du gardien du phare et d'Emmanuel. Ces deux personnages sont la mémoire des incidents qui scandent l'existence de l'île. La description des parcours des nuages les rend semblables aux vols des oiseaux: "Un orage avait traversé l'île et l'avait dépassée" (ibid.: 84) ou à un coup de pinceau insolite: "le regard fixé sur la mer que violaçait le passage d'un lourd nuage solitaire en transit au-dessus de l'île" (ibid.: 81 et 211). Le violet est précisément la couleur du passage, symbole notamment de l'entre-deux rêve-réalité. Dans cette île où tout n'est qu'attente et contemplation, la chape de nuages introduit une nouvelle temporalité, celle qui suit la mort, celle d'un après-coup. L'image du deuil en cours est associée de façon récurrente aux nébuleuses qui proviennent de la mer du Nord: "Il [le vent] repoussait le mercure au fond de son baromètre et emmenait en transhumance des troupeaux de nuages qui, traversant l'horizon d'un bord à l'autre de la clôture ronde de l'horizon, endeuillait l'île sous une lumière grise" (ibid.: 149). Le paysage dépeint prend le contre-pied des clichés traditionnels relatifs à I'horizon, en raison de la présence des nuages: encerclée par la mer, l'île n'est plus un espace ouvert sur l'immensité. Le thème de la mort ressortit au verbe de la proposition relative et à l'épithète de couleur qui qualifie le mot "lumière" et qui, du fait de la préposition qui précède le groupe nominal, réifie 
ce halo, implicitement identifié à un voile. Cette métaphore est explorée dans les pages suivantes (cf. notamment l'expression "des nuages ont commencé à recouvrir l'île" [ibid: 191]).

A l'instar des oiseaux, ces nuées ont le pouvoir de posséder toute l'île d'une manière tangible. L'état létal, comme figé, qui affecte l'île contamine le phare: "L'île était dans l'ombre, et la cabine, dont aucun rayon ne venait plus frapper les vitres, semblait morte" (ibid:152). Or, ce bâtiment est emblématique du regard de l'Homme, de sa volonté de dominer l'espace environnant. Sa puissance est remise en cause par l'astre solaire qui échappe à tout contrôle: "Le faisceau de soleil avait glissé hors de l'île, à la façon d'un projecteur déréglé dérapant de la scène, et donnait à la mer un éblouissant éclat mécanique qui venait s'éteindre le long du rivage" (ibid.: 153). La comparaison au matériel scénique identifie l'île à un plateau de scène abandonné. L'image donne explicitement à entendre que les acteurs du drame insulaire sont les éléments. L'adjectif "mécanique" qui précise le caractère aveuglant de cette luminosité relève du champ de la culture et non de la nature. Le Verbe qui les magnifie est en filigrane une ultime protestation contre la vulnérabilité des Hommes ainsi mise en relief. A la fin du récit, le motif de la mort trouve son aboutissement dans l'image de l'île noyée sous l'averse: "La pluie s'était mise à tomber, [...] noyant la crique, noyant l'île et la mer, noyant les nuages" (ibid.: 208). Cette eau vive et éphémère, provenant de la mer, fait partie de l'espace nordique ${ }^{6}$. Elle partage avec lui cette aptitude à s'auto-générer, qui prend parfois l'aspect d'une autodestruction.

Néanmoins, la mer lodsienne n'est pas romantique: jamais démontée, jamais personnifiée, sans profondeur inquiétante, elle annonce plus volontiers l'autre côté du continent. Du haut d'une crête, la mer marque la fin de la course: "C'est comme une façon de les [le personnage éponyme et son grand-père] prévenir que de l'autre côté c'est le vide, avec la mer au-dessous" (Lods, 1991: 109). L'air sépare la mer de la terre. Surface implacable, elle est confinée aux basses altitudes. Jamais haute, car basse et guère éloignée des rivages. Une analyse rapide et approximative pourrait considérer la mer comme une manifestation de l'érotisation de l'espace, qui opposerait l'île phallique à la mer horizontale, allongée. Mais la géographie lodsienne n'est pas célinienne ${ }^{7}$. La hiérarchie de l'espace, affective, procède d'une perspective logique personnelle, qui ressortit davantage à l'image du puits artésien sur laquelle repose la structure de certaines œuvres de Claude Simon (1997a, b). Elle s'en distingue cependant, dans la mesure où elle résulte d'une intrication de la mémoire, du lieu et de l'affect. En effet, l'appréhension lodsienne de l'espace

\footnotetext{
${ }^{6}$ Dans l'ensemble des récits lodsiens, l'espace nordique est constitué de l'île de la mer du Nord évoquée ici (Sven, 1991), mais également d'une lagune (Le Silence des autres, 1973; La Part de l'eau, 1977), de la ville de Prague et du grand nord (Quelques Jours à Lyon, 1994).

7 Cf. la comparaison de New York "debout, raide, pas baisante du tout" aux villes françaises "couchées" (Céline, 1989: 237).
} 
accorde une part conséquente à l'espace, quand Claude Simon, à la suite de Proust, adopte une démarche avant tout mémorielle: l'objet envisagé (le pavé, le revolver) se confond avec la mémoire de l'objet, n'existe qu'au travers de cette mémoire.

La mer apparaît alors comme une manifestation du lieu du lieu, une métaphore du lieu de l'espace: elle est parce qu'elle est, quitte à n'être plus qu'un aveuglement. Ainsi en est-il de la mer aveuglante du Bleu des vitraux dans le souvenir de Yann Toulec. A la fin du récit, il s'imagine en effet le trajet de la voiture partie en trombe de la propriété de Hell-Bourg, dans laquelle son père le retient captif. Une phrase nominale évoque, à l'instar d'un instantané, la surface de l'eau impénétrable: "La phosphorescence de l'océan" (Lods, 1987: 175). L'élément marin peut ainsi devenir indistinct pour l'œil qui l'observe en raison d'une visibilité excessive. II oppose au regard une surface solide, uniforme et immobile. Dans les premières pages de Quelques jours à Lyon, l'inaccessibilité de la mer est le fait non seulement de la distance et de la grille qui séparent, mais aussi de l'aveuglement dû à la luminosité: "II [Romain] était resté devant elle [Hélène, sa femme], contemplant la route à travers les barreaux, et, au-delà, l'étendue éblouissante de l'océan au bas de la montagne" (Lods, 1994a: 22). Dans le face-à-face avec l'épouse trompée, la mer devient l'objet du point de fuite aveuglant.

La mer aveuglante est aussi associée à une infidélité, dans Le Bleu des vitraux, à la "trahison" de la mère qui regagne la métropole définitivement. Yann refuse autant que faire se peut son souvenir: "Il y avait aussi ma façon de [...] me tenir loin des bords de mer parce que les éblouissements de l'océan Indien me brûlaient encore les yeux" (Lods, 1987: 22). La cécité semble à la fois le fait du reflet de la lumière sur les flots et des résistances affectives. Malgré le regard en surplomb, la mer ne se voit pas. Elle s'impose à la perception comme une entité qui forme une surface unie. II est difficile en effet de se déplacer dans l'île lodsienne sans rencontrer la mer. L'itinéraire imaginaire qu'emprunte Yann après le départ des visiteurs constitue une fuite aussi improbable que cette mer invisible, car éblouissante. La mer aveuglante coïncide avec l'île inaccessible. En témoigne également cette occurrence extraite de Quelques Jours à Lyon: "Romain revit l'éblouissement de l'océan Indien, les palmes moulinant le ciel sous l'alizé" (Lods, 1994a: 251). Au moment où le personnage doit répondre à l'hôtesse de l'agence de voyage quant à la destination de son vol, l'île s'impose à son esprit sous la forme d'une brève image d'Épinal, favorisée par une photographie affichée au mur. L'écran marin aveuglant coïncide avec le projet qu'il ne peut plus envisager. La mer ne saurait donc être réduite au statut de composant d'un paysage ou d'une géographie imaginaire: elle participe non seulement de l'événement, mais aussi de la construction des personnages, dans la mesure où elle est susceptible de représenter cette "essence de l'être qui est d'être encore là où il manque" (Blanchot, 1998: 340). 
Le paradoxe de la mer réside pour l'essentiel dans cette domination qu'elle ne cesse d'imposer à la qualité du regard qui n'est au-dessus d'elle que par sa seule localisation: elle soumet l'être qui l'observe depuis les hauteurs. Le chronotope crépusculaire en offre une autre illustration. La narration associe souvent l'océan Indien au crépuscule vespéral, de sorte que sa manifestation participe à la scansion des journées. Cependant, cette combinaison de la mer et du soir dépasse la fonction d'indice temporel: elle coïncide avec un événement porteur d'un bouleversement profond. Ainsi, dans Le Bleu des vitraux, l'incandescence du coucher de soleil sur la mer complète-t-elle la scène emblématique de la glace: sous prétexte de manger une glace, René Toulec éloigne son fils de la maison afin de savoir si celui-ci est affecté par la crise que traverse son couple. Le père, embarrassé, aborde le sujet en évoquant un changement survenu dans l'attitude d'Anne-Sylvie. Père et fils sont attablés, tandis que "la toile de fond" semble exprimer leurs colères rageuses respectives: "l'on dirait que l'horizon a pris feu sous le tir des noirs canons de bronze ${ }^{8}$ alignés sur la promenade et pointés vers l'océan" (Lods, 1987: 125). Le crépuscule marin se substitue aux mots qui ne sont pas prononcés.

Dans Sven, cet entre-temps accompagne toute la fin du récit. L'une des premières occurrences rappelle la mort du gardien du phare: "Mais personne maintenant n'allumait plus de feu pour réchauffer la mer du Nord quand le jour était sombre" (Lods, 1991: 217). La personnification que suppose le verbe "réchauffer", bien qu'abandonnée aussitôt, rend hommage au vieil homme et induit implicitement une osmose entre celui-ci et l'élément. Dans l'avant-dernière page, l'opposition de la couleur du ciel au-dessus de la mer et audessus de l'île annonce le départ de Sven: "Un rayon jaune et rasant vola d'un trait sur la mer, dorant l'écume des vagues et le sable, auréolant les oyats des dunes, alors qu'audessus de l'île la chape du ciel restait lourde et plombée" (ibid.: 223). L'horizon qui reprend le personnage éponyme est lumineux (ibid:: 218). Les propositions participiales qui le caractérisent se déploient en un rythme ternaire qui contraste avec les deux attributs baudelairiens qui qualifient les nuages sur l'île. Cette miniature expressionniste annonce la séparation du point de vue de celui qui reste. La page suivante lui offre une suite en une phrase-paragraphe: "Le soleil disparut derrière l'horizon, et le bref incendie qui avait enflammé les nuages au-dessus de la mer s'éteignit presque aussitôt" (ibid.: 224). Le dernier rayon de soleil s'efface peu de temps après les derniers traits de la silhouette de l'adolescent. Le verbe "s'éteindre" rend à la mer sa substance liquide et réinstaure le duel stéréotypé de l'eau et du feu.

Dans Quelques Jours à Lyon, le recours au crépuscule marin répond à une dramatisation inverse, qui revêt une tonalité comique plutôt que tragique. II apparaît en effet

\footnotetext{
${ }^{8}$ L'histoire de ces canons et leur convocation dans la fiction sont respectivement retracées et analysées in Gendre, 2007: 351 et 359.
} 
dans une scène cocasse, dans le rêve du retour du père post mortem, dans l'île des années 1950: Simon Rivière, écrivain vieillissant, pédale sur son ancienne bicyclette d'enfant, dans les rues du vieux Saint-Denis. Le rougeoiement de la mer accompagne la folle équipée: "Sur sa droite, à côté du cap Bernard ${ }^{9}$, le soleil commençait à descendre vers l'océan, la nuit ne tarderait pas à venir" (Lods, 1994a: 65). II signale le retour tardif à la maison et préfigure le blâme de la mère. Cette anecdote de l'enfance rappelée par le récit du rêve reste solidaire des autres occurrences du crépuscule marin. Celui-ci est toujours peu ou prou synonyme d'une prise de conscience tardive qui génère un sentiment de culpabilité.

Bien que localisée en contrebas dans cette géographie subjective, la mer échappe à toute tentative de contrôle. La connivence de la mer et du ciel, par sa correspondance avec les après-coups, achève de montrer que la fonction de l'élément marin ne saurait être réduite à la constitution d'un décor. Si la mer apparaît dans une relation d'euphorie avec la conscience des personnages ou des narrateurs, elle domine souvent la subjectivité et le regard qui la contemplent, si bien qu'elle joue un rôle fondamental dans ce qui est doté ou dépourvu d'existence pour le sujet. Elle révèle autant les choses que le sujet à soi-même. L’ambivalence étudiée ci-après éclaire de façon majeure cette épiphanie essentielle.

\section{L’avalage et la fonction génésique de la mer}

L'appréhension de la mer dans les récits lodsiens relève d'un retranchement d'être qui est notamment le fait du motif des "entrailles" qui traverse un certain nombre de ses évocations. L'élément est en effet volontiers identifié tantôt à une bouche, tantôt à un ventre géant. Existe-t-il un adversaire susceptible de contrer cette oralité? Quel éclairage nouveau apporte la mer de sable septentrionale sur cette ambivalence tropicale?

Dans la représentation que se fait le narrateur du Bleu des vitraux de l'île de son enfance, la mémoire revisite un instant le mythe de Jonas en prêtant à la mer un caractère viscéral. Le gigantisme de cette vision est éloquent. La mer met l'île au monde: "Alors, seul, divinement seul, je pouvais enfin regarder l'île naître de la mer dans son collier d'écume" (Lods, 1987: 22). La mémoire de l'île tropicale saisie dans sa globalité semble ici puiser dans le souvenir de nombreuses vues aériennes de l'île. Cette image poétique de la naissance achève la personnification de l'île en la féminisant: le rempart de coraux et de roches provoque un cercle d'écume (le "collier"), dont l'île devient le centre. Cette déhiscence de l'île, à la fois imaginaire et mémorielle, préfigure la vision qui s'impose à Emmanuel du personnage éponyme de Sven qui "semblait sorti de la mer sur son cheval" (Lods, 1991: 218). L'isotopie emprunte ici au fonds archaïque des peuples marins qui attribuent à

\footnotetext{
${ }^{9}$ Cf. l'analyse de la symbolique de la couleur rouge et du feu dans la représentation récurrente du crépuscule marin depuis le cap Bernard. In: Gendre, 2007: 284-287.
} 
l'élément aquatique une fonction matricielle, souvent doublée d'une force destructrice. L'imaginaire lodsien n'exclut pas cette ambivalence. En effet, le ventre marin peut également engloutir, comme le montre l'évocation de "la lente immersion de Bourbon dans l'océan" (Lods, 1987: 160). Le ventre-bouche s'associe dans Sven avec "la longue langue brumeuse du continent étalée sur le bleu de Prusse de la mer" (Lods, 1991: 64). La paronomase que réalisent l'adjectif qualificatif et le nom auquel il se rapporte ("longue langue") et à laquelle fait écho l'allitération de consonnes liquides, parachève la suggestion d'un continent reptilien. A l'échelle de ce monde-bouche continental et maritime, l'être humain disparaît.

A la différence du continent, l'île est continuellement mangée par le ventre bleu. Le travail de l'érosion des parois du littoral se caractérise par sa violence: "les vagues de l'océan Indien broient ${ }^{10}$ indéfiniment les galets du Barachois..." (Lods, 1987: 186), "[i]l se promenait le soir le long du Barachois, avec, d'un côté, le fracas des rouleaux déferlant sur les galets du rivage, de l'autre le défilement sans fin des voitures sur la route du littoral" (Lods, 1994 a: 117), "le déferlement des vagues en bas du parapet" (ibid.: 155). Le terme "déferlant" doit être saisi dans son acception maritime, et non comme un adjectif galvaudé: les vagues sont perçues comme une agression par l'insulaire lodsien. L'île n'en paraît que plus fragile. Sven donne à lire deux descriptions d'une Réunion menacée de disparition. La nature reprend ses droits sur les velléités de civilisation: le cirque, île dans l'île, est gagné par la végétation, tandis que la mer entame la base de ses remparts: "ce cirque désert où la forêt envahissait peu à peu les plantations abandonnées, cette île qui semblait rétrécie au point que maintenant l'océan venait battre au pied des montagnes qui en ceinturaient l'intérieur..." (Lods, 1991: 62). Synopsis apocalyptique qui envisage, de l'intérieur vers l'extérieur, du centre à la périphérie, l'annulation de toute la dimension protectrice de l'espace insulaire. La polysémie du terme "battre" superpose le champ de la guerre et celui de l'art culinaire. L'infaillibilité de l'élément est totale. La toute-puissance de la mer est également illustrée par une comparaison qui emprunte au corps de pierre du golem mythique: "Tout en bas de la paroi qui tombe comme un drap de pierre, un long cordon d'écume ronge les rochers" (ibid.: 109). Le verbe "ronger" animalise le ventre-bouche marin.

Son infaillibilité et sa violence sont aussi le fait des sons qu'il produit. La plupart des évocations recourent à la synesthésie et mêlent l'ouïe au tactile. Le choix des termes (cf. les mots en italique ci-dessous, soulignés par nous) révèle une intensité croissante au fil de l'œuvre: "l'éternel grondement râpeux ${ }^{11}$ de l'océan Indien sur les galets du littoral" (Lods, 1987: 147) devient dans cette description plus tardive "l'océan dont on entend le grondement des vagues explosant contre les rochers au bas de l'à-pic" (Lods, 1991: 50). Dans cette

\footnotetext{
${ }^{10}$ Tous les mots en italiques, dans les citations de ce paragraphe, sont soulignés par nous.

${ }^{11}$ Tous les mots en italiques, dans les citations de ce paragraphe, sont soulignés par nous.
} 
occurrence-ci, l'île est envisagée par le personnage de l'enfant, et non par le narrateurpersonnage Emmanuel (cf. ibid.: 62). Les éléments du paysage retenus, le cirque et l'océan, sont évoqués dans un ordre inverse, selon que l'île est appréhendée au travers du souvenir du narrateur (la vision globale de l'espace va de l'intérieur vers l'extérieur de l'île) ou par le personnage éponyme qui parcourt un sentier du cirque (de l'extérieur inquiétant vers le refuge intérieur). Le heurt des galets connaît la même intensité crescendo. Permanent, sur le littoral tropical, il tend à se substituer au silence: 'Là [sur le rivage] il n'y avait d'autre bruit que celui de la mer qui roulait les galets" (Lods, 1980: 44). En revanche, l'absence de toute distraction sonore le rend redoutable: "Le silence s'installe à nouveau, qui rend insupportable le raclement des vagues sur les galets de la côte depuis le cap Bernard jusqu'à SainteSuzanne" (Lods, 1987: 115). La synesthésie produite par le lexème "raclement" est, à l'instar des verbes et des groupes nominaux soulignés ci-dessus, de connotation péjorative. Elle est le fait d'une appréhension subjective de l'élément marin. Le déplacement d'affects qu'opère l'insulaire tend à faire de la mer une autorité qui menace l'île, l'inquiète et la blâme.

Mais quelles forces parviennent à contraindre le monstre marin? C'est principalement grâce à l'économie coloniale ${ }^{12}$ que l'homme tente de lui résister et se montre capable d'avaler quelque peu l'avaleuse, quand l'averse tropicale et le vent constituent ses principaux adversaires naturels. La puissance de la mer se manifeste pour l'essentiel, par la métamorphose qu'elle parvient à opérer sur les sites jusque-là régis par les seules lois de la nature. L'économie, en tant qu'ensemble des activités qui ont trait à la production, à la distribution et à la consommation, impose une répartition utilitaire de l'espace insulaire, fondée sur le critère du rendement.

La Morte saison est le seul récit lodsien qui décrit ce phénomène comme lié au régime colonial. De retour dans l'île de son enfance, Martin, le narrateur-personnage, prétexte une enquête sur un homme qui est en fait son père, en se faisant passer pour un écrivain. Son investigation l'entraîne dans la propriété d'été des Villette, sur les traces de ses amours enfantines pour Eléonore qu'il recouvre sous les traits de la fille bannie de cette dernière, Marieka. Dans la séquence XV du premier volet, l'implantation des Villette sur le littoral est évoquée dans le paragraphe qui suit le résumé relatant le rituel de la paie des ouvriers de Quartier Français. Ces derniers vivent sur ces "terres des hauts, moins productives que celles du littoral, plus difficiles d'accès pour les camions" qui "avaient été peu à peu abandonnées et tombaient en friche" (Lods, 1980: 99). L'infléchissement de l'occupation de l'espace aux priorités économiques est explicite. Cette répartition spatiale inverse la verticalité de la pyramide sociale: "La famille des Villette avait suivi cette évolution et avait conquis le littoral" (ibid.). Les classes ouvrières habitent les hauts, tandis que les

\footnotetext{
${ }^{12}$ La Réunion fut colonie française jusqu'en 1936, année de la départementalisation de l'île.
} 
propriétaires de domaines occupent le littoral. Le souci de l'accessibilité préside à cette répartition spatiale. Ainsi les centres névralgiques de l'économie sont-ils à l'extérieur de la périphérie des cirques, en bord de mer. Peu à peu, l'économie dénature le rivage et écarte des préoccupations humaines le travail de l'avaleuse marine: "La canne et l'usine à sucre ${ }^{13}$ sur le littoral étaient apparues avec monsieur Villette ${ }^{14 "}$ (ibid.: 200). Dans ce récit, les descriptions du littoral des années 1950 le présentent invariablement comme le fait des activités économiques dont il est le théâtre: "Sur le littoral, les feux d'artifice des flamboyants devaient commencer à s'allumer dans les cours et le long des boulevards, et les petits marchands des hauts à aligner au bord des trottoirs leurs paniers de mangues ou de letchis" (ibid.: 141). Le soleil de l'aube se reflète dans les feuilles des arbres tropicaux: le premier sujet de la phrase, métaphorique, réalise une alliance confiante de la nature et de la culture. Le groupe nominal "feux d'artifice" fait allusion aux fleurs rouges des flamboyants. Les "cours" (intérieur) et les "boulevards" (extérieur) signalent des infrastructures urbaines de type européen. Les habitants des hauts y ont leur place et leur heure, comme réservées, pour vendre les surplus de la récolte des jardinets volés à la montagne. Ainsi les déplacements humains sont-ils également soumis aux exigences d'ordre économique, toutes classes sociales confondues.

Les allusions à la présence de l'élément marin dans la cité tropicale tendent souvent à briser avec l'angélisme des littératures exotiques et coloniales. Ecrit sept ans après $\mathrm{La}$ Morte Saison, Le Bleu des vitraux évoque un littoral déprécié. La Veuve Toulec le condamne en lui prêtant une insalubrité qui a longtemps été prêtée aux villes portuaires, comme le montre cette parenthèse: "(les femmes du littoral, qui vivent dans un climat torride et paludéen, ont un lait moins sain que celles qui respirent l'air pur des montagnes)" (Lods, 1987: 42). Son fils, René, reproche au chef-lieu du jeune département "sa chaleur excessive, ses fièvres, la vie provinciale" (ibid.: 88). L'adjectif "provinciale", en raison de la répartition spatiale (province / capitale) qu'il suppose, indique que la critique repose implicitement sur des critères métropolitains. Quant au terme "fièvres", il peut être employé comme une métonymie du paludisme, dans la mesure où il désigne l'un des symptômes principaux de cette affection, de même qu'il peut désigner dans un emploi figuré l'exaltation suscitée par la vie nocturne. Saint-Denis est toujours perçue comme un ensemble: "Là-bas, sous l'ombre immense du cap Bernard, éteintes, les maisons de Saint-Denis" (ibid.: 78). L'éclairage urbain

\footnotetext{
${ }^{13}$ Substitut lexical qui désigne Quartier Français, le producteur le plus important de sucre de canne de l'île de La Réunion, dans les années 1950.

${ }^{14}$ Monsieur Villette est une représentation romanesque de René Payet, directeur de Quartier Français, principal adversaire politique de Paul Vergès, l'actuel directeur de la région Réunion. A peine débarqués dans l'île, Jean Lods et sa mère ont été recueillis par Madame Payet. Jusqu'à ce qu'il quitte définitivement l'île pour la métropole, l'auteur passait ses vacances avec la famille Payet, dans leur domaine de Mare à Citrons. Cf. pour les données biographiques relatives à Jean Lods: Annick Gendre (2007). "L'Espace d'une vie, biographie-fiction de Jean Lods" [en ligne]. In: La Part de Lods [consulté le 29/12/2008]

<URL: http://dunerivelautre.free.fr/index_fichiers/page10.htm>.
} 
retient volontiers l'attention de l'observateur. Quant aux infrastructures du littoral, elles ne concernent que le réseau routier: "De temps en temps passe encore l'étoile filante d'une voiture sur la route du bord de mer" (ibid.) et "ll change une nouvelle fois de direction un peu plus loin avant de retrouver le bord de mer" (ibid:: 122), pour ne retenir que ces exemples. L'intérêt récurrent du narrateur pour la route qui suit le littoral est à la mesure du problème obsédant de l'accès aux terres intérieures de l'île.

Or, la mer réapparaît au hasard des tournants de la route, à chaque fois dans des descriptions behavioristes dans lesquelles le narrateur dénonce, en adoptant le type interrogatif, son ignorance quant aux réflexions de son père. L'interrogation achève d'établir une relation entre la mer et le champ des certitudes et des incertitudes. Le jeune zoreil s'inquiète des raisons qu'il va pouvoir invoquer auprès de sa mère pour expliquer son retard (un incident, qui, du fait de sa récurrence dans l'œuvre doit être rattaché à un réseau de thèmes qui fait motif), tandis que le narrateur captivé par le spectacle sensuel du bain vespéral d'Anne-Sylvie se demande: "Avait-il ces images en tête, René Toulec, en rentrant à Bois-Rouge par la route qui suivait le littoral?" (ibid.: 38). Artifice romanesque pour relancer la narration? Pas seulement: l'interrogation accepte l'ignorance et autorise le fantasme. A l'instar du chantier de la lagune des deux récits liminaires de l'auteur (Lods, 1973 et 1977), toutes les tentatives de contraindre le grand œuvre de la mer sont vaines. Cette impuissance de l'Homme face à l'élément habite l'insulaire. C'est pourquoi, semble-t-il, pour donner libre cours à la colère vaine que lui inspire le carcan des obligations dévolues à son milieu social, René Toulec anticipe et rejoue sur le bord de mer le scénario de la mise en échec: "A quoi pense-t-il un peu plus tard quand il se retrouve sur la plage, et que sa main, saisissant un galet, le lance avec emportement dans une trajectoire qui s'achève misérablement à quelques mètres du rivage?" (Lods, 1987: 46). Le galet n'est plus roulé, mais livré à l'avaleuse. Aucun défi n'est lancé à l'élément. Celui-ci se fait le théâtre du renoncement.

Le seul adversaire naturel que connaît le gigantesque ventre bleu s'invite sur la scène romanesque avec l'averse tropicale. Son composant essentiel, l'eau, se déversant avec la violence dévolue au climat subéquatorial, est représenté comme capable de la mettre à mal: "La pluie s'était mise à tomber, ruisselant sur le visage de l'homme, noyant la crique, noyant l'île et la mer, noyant les nuages" (Lods, 1991: 208). Le rythme ternaire scandé par la répétition du participe présent "noyant" parachève la description de la toutepuissance que lui attribue le regard insulaire. En filigrane, l'eau retourne à l'eau, comme la poussière à la poussière: le texte revisite la parabole biblique. Le partage des eaux n'opère plus. De longue date, l'île de La Réunion est divisée en deux zones distinctes par leur climat: la "côte au vent", qui suit le littoral nord-est, et la "côte sous le vent", le long du littoral sudouest. La première correspond à l'aire la plus humide. Dans Mademoiselle, l'ultime rencontre d'Eric Feuguet avec le fantôme de sa mère, décrit explicitement la concurrence de la pluie 
avec la mer quant à l'envahissement des terres: "les nuages, qui montent depuis la 'côte au vent" [sic], "nappent de brouillard le plateau et remplissent les cuvettes des vallées" (Lods, 1994b: 34). Cette eau rivalise avec la mer non seulement en raison de son incursion dans les terres, mais également parce qu'elle rend certaines zones de l'île plus clémentes à I'habitat humain. Ainsi ces deux zones témoignent-elles d'un découpage de l'espace, comme en témoigne l'implantation des Toulec "sur les terres humides de la Côte sous le vent [sic]" (Lods, 1987: 43). La présence ou l'absence du vent participe de l'identité du lieu, sur terre comme en mer. L'élément éolien semble l'un des principaux composants marins qui parvient parfois à réguler les appétences océanes.

Dans les fictions de Jean Lods, son évocation rare le rend emblématique: il participe de l'événement dans lequel la narration le convoque. Ainsi, un instant avant la scène de la glace: "Le vent qui souffle de l'océan gonfle la veste de René au moment où il ouvre la portière et sort" (ibid:: 124). Le récit prend le temps de préciser sa provenance, dans la subordonnée relative. Bien qu'elle soit du point de vue géographique et climatique évidente, elle a une fonction romanesque. Elle annonce le départ de la mère, le paquebot, l'angoisse d'abandon et le narcissisme blessé. Ces évocations sont d'autant plus précieuses que le vent, à l'image d'un sculpteur, restitue à la mer une forme et une consistance: pour un temps seulement, la narration rompt avec le motif de la surface et de l'écran. Ainsi dans Sven et dans Quelques Jours à Lyon peut-on lire: "Le vent était tombé, mais la mer était grosse" (Lods, 1991: 19) et "Le vent était encore fort après le passage du cyclone qui avait effleuré l'île, la mer lourde et bosselée" (Lods, 1994a: 124). La forme comme la consistance ("grosse, lourde, bosselée") s'avèrent indissociables ici de la pesanteur.

Autre marine: la tempête. Seul l'archipel néerlandais en est le théâtre. Sa violence est à la mesure de la bataille que se livrent les éléments. Sven s'ouvre sur la détresse d'un navire en prise avec le déchaînement, au large: "Le cargo demeurait insaisissable dans la tempête de la mer du Nord" (Lods, 1991: 9). On reconnaît le complexe de Jonas: l'avalage de la mer et du ciel fusionnés se manifeste aux deux vigies du phare. De plus, il est probable que la dramatisation de son évocation soit le fait d'une réminiscence inconsciente de certaines toiles hollandaises, comme le donne à penser le tableau suivant. L'autoscopie (le peintre évolue lui-même dans la toile qu'il réalise) fait du narrateur-personnage un avatar de l'île: il subit le même assaut que sa rive. L'élément force son ouĩe, tandis que la surface liquide devient subitement tactile: "très loin, mais se rapprochant à mesure, on entendait le bélier brutal de la mer heurtant de front le rivage. La tempête me frappa de plein fouet quand je débouchai sur la plage" (ibid.: 124). La métaphore vive de l'arme de guerre ("le bélier") cède la place à l'image lexicalisée "de plein fouet". Le verbe "frapper" invite à lire le terme "fouet" au sens propre. Lequel, de la mer ou de l'élément éolien, domine l'autre? Lequel des deux instrumentalise la force de l'autre? Si le texte ne permet pas de trancher en faveur de 
l'un ou de l'autre, la spatialité qu'il met en scène se trouve enrichie du fait de la présence de ces deux frères ennemis.

Mais l'écriture lodsienne s'empare également d'une autre mer, celle-ci de sable, offerte par les dunes septentrionales. D'une part, elle constitue le composant essentiel de l'île nordique, celui qui permet de l'identifier comme telle, d'autre part, elle s'impose comme emblématique de l'ambivalence qui associe l'incorporation orale et la reproduction: non seulement la perception dont cette autre mer est l'objet prolonge l'élément marin, mais ses convocations font intervenir simultanément l'avalage et la fonction génésique. L'horizontalité des dunes en fait un élément féminisé sans lequel rien de ce qui est déhiscent sur l'île des Wadden n'apparaîtrait, tels que les insulaires exclusivement de sexe masculin, ainsi que le phare. Elle est la matérialité de l'île, telle que le narrateur-personnage se la représente: "Ce paysage de dunes, où l'attend l'homme au loden vert, si longtemps incertain, si longtemps limité aux moutonnements entourant comme un décor de théâtre la silhouette malmenée par le vent, s'est développé chaque jour depuis sa première apparition" (ibid:: 190). Le terme "moutonnements" fait référence par métaphore aux dunes, à la suite d'une analogie de forme que le sable partage avec les nuages ainsi qu'avec l'écume. II est fréquemment employé dans l'écriture lodsienne à cette fin. Les dunes sont investies en tant que théâtre de l'attente où s'élabore la mise en scène rêvée de l'arrivée de Sven. Elles se déploient parallèlement au scénario onirique et, de même que pour les oiseaux, leur textualisation participe à la représentation de l'acte de création, au point de s'amalgamer parfois complètement à cette dernière.

Les dunes sont la chair de l'île que le narrateur se plaît à personnifier. Dès les premières pages du récit, tandis qu'il observe les photographies d'Anne que lui montre le gardien du phare, il ne peut s'empêcher de comparer ainsi l'île nordique à l'île tropicale: "Avec les épaulements des dunes se dégageant à peine du tissu de la brume, elle semblait l'image érodée de l'autre île, celle, volcanique, de mon enfance" (ibid.: 19). Alors que le brouillard habille les rondeurs de son sol de sable, La Réunion reste avant tout minérale et anguleuse. On retrouve l'image du vêtement féminin: "le voile de sable arraché aux dunes" (ibid.: 207). II coïncide avec "l'univers tourbillonnant et gris" dans lequel se trouve Emmanuel, qu'il s'agisse de l'île sous le vent ou de son état intérieur. L'anatomie de l'île septentrionale est ainsi répartie, quelques pages plus loin: "Le sentier suivait encore un moment la digue de sable qui formait l'arête centrale de l'île, puis l'abandonnait pour plonger entre les dunes" (ibid.: 33). Le verbe "plonger" identifie implicitement les dunes à une mer de sable accueillante. Celles-ci s'opposent à l'ossature de la digue. Incontestablement, en elles réside ce qui fait de l'île un espace accueillant. Cela ressortit à la toile de fond que les dunes offrent à tout ce qui fait saillie. II en est ainsi du phare: "la flèche de la tour se dressait au-dessus des dunes" (ibid.: 209). Le substitut lexical employé achève de lui donner un caractère 
phallique dans cet espace féminisé. Les nombreuses indications de lieux de passage qu'elles abritent dans leurs rondeurs témoignent également de cette capacité d'accueil. Les déambulations d'Emmanuel qui pénètre et parcourt sans cesse leurs courbes contribuent à cette érotisation du paysage insulaire qui les place sous le sceau du féminin.

Enfin, cette mer de sable est l'unique élément spatial de l'île qui est sonore, trait qui lui donne consistance et épaisseur. Cette matérialité se manifeste au contact de l'eau: "La pluie, s'abattant sur l'étendue des dunes, la noyait sous un bruit immense" (ibid.: 219). Seul le son produit par ce contact est capable de "noyer" (ce verbe fait de l'espace un être animé) l'île et corrobore l'immuabilité des dunes qui font l'objet d'une appréhension fantasmatique, comme le montre la textualisation de l'écriture qu'elles illustrent. Rien d'étonnant à ce qu'elles confèrent à l'île sa solidité et, en dépit de l'érosion qu'elles subissent naturellement, un caractère immuable. Elles demeurent intactes d'être sans cesse renouvelées. Sans doute est-ce pour cela que le narrateur les désigne comme des "barrières de sable" (ibid.: 124). Elles protègent incontestablement, mais à la différence de l'île de La Réunion, elles n'enferment pas. L'île nordique est une forteresse mais de laquelle le narrateur-personnage ne désire point s'échapper. Ce bastion de sable a, en effet, la particularité d'offrir un espace mobile qui prend l'aspect d'un être vivant. Cette mobilité est le fait de la variété de ses formes. Ainsi "le cordon de dunes" (ibid.: 211) succède-t-il au "plateau de dunes" (ibid.: 210). La surface donne lieu à une ligne et fait écho à l'image du texte-paysage, apparue dans les pages précédentes. La polysémie du mot "plateau" permet ici la superposition de ses acceptions: il désigne à la fois la surface géographique et l'espace scénique. Les dunes apportent des lignes, mais aussi des courbes à la surface de l'île. Elles invitent à un décryptage: "La camionnette [...] disparut en contournant la longue virgule de la côte que bosselaient les dunes" (ibid:: 83). La métaphore qui les dote de leur rondeur repose sur cette analogie avec un signe de ponctuation. L'île devient un espace-texte. Son observation et sa description, une lecture.

L'avalage et le pouvoir génésique de la mer impliquent l'un et l'autre l'originel, en tant qu'événement primordial et en tant que phénomène actif. Les deux adversaires, naturel et humain, n'opposent qu'une résistance dérisoire à cette incorporation et à ces délivrances répétées, qu'elles soient créatives ou destructrices. L'éphémère de sable sans cesse recommencé des dunes en offre plusieurs déclinaisons et s'impose en tant qu'accueil des possibles. Possibles de l'histoire et possibles de l'acte d'écriture.

\section{La référentialité textuelle de la surface marine}

Le singulier partage dont rend compte l'élément marin au sein de la spatialité lodsienne se révèle autant un instrument narratif qu'une manifestation polyphonique. La 
richesse sémantique du terme partition apparaît alors tout à fait comme la plus adéquate pour rendre compte de l'épiphanie marine. Cette polyphonie qui associe les personnages au narrateur et à l'auteur se donne à lire dans le réseau de références multiples dans lequel est convoquée cette mer imaginaire. Comment cette polyphonie est-elle orchestrée? Si la mer génère la circonférence de l'île, elle participe pour cette raison à la représentation de l'île en tant qu'emblème universel du centre spirituel primordial ${ }^{15}$. Cependant, malgré son ventrebouche, la mer lodsienne n'est pas profonde ${ }^{16}$. Quelles sont les incidences de cette surface marine, de ce qu'il convient d'appeler "miroir du monde", sur les qualités du centre insulaire? Pour les apprécier, il importe d'envisager la référentialité de la mer telle qu'elle apparaît dans les mimétismes qui affectent l'élément représenté.

Avant d'être liquide, la mer lodsienne est plastique. Le regard se pose sur sa surface sans parvenir à la scruter, à en saisir un seul détail. Sa consistance réside dans cet écran qui la manifeste. A la différence du continent, la mer-écran arrête le regard, lui fait obstacle, si bien qu'il signale le fond du vide. Pourquoi le narrateur se plaît-il à ce point à donner à percevoir l'élément marin dans ce qu'il a d'invisible? Le temps que les personnages consacrent à contempler cette fin du vide est remarquable, particulièrement dans Le Bleu des vitraux et dans Sven. Dans ce dernier récit, le phare joue un rôle important dans cette contemplation. A l'intérieur, l'île est identifiée par son gardien à un navire: "— L'île! Elle se prépare à appareiller vers le nord. Elle est déjà orientée dans cette direction, et devant elle la mer est libre jusqu'au pôle" (ibid.: 32). La métaphore filée ("appareiller vers, orientée, libre") en témoigne. L'élément marin devient une route, comme l'indique le personnage, en revisitant l'expression toute faite la voie est libre.

Dans la vision délirante du vieux gardien du phare, l'attraction du pôle annonce celle que Romain éprouve dans Quelques jours à Lyon. La cabine du phare favorise cette analogie. L'isolement insulaire explique aisément les fantasmagories dont cette cellule ouverte fait l'objet. L'investissement subjectif du paysage marin est aussi sensible dans les marines nocturnes. Par exemple, Emmanuel projette d'observer, durant les nuits à venir, la noirceur des flots depuis cet observatoire stratégique. Remarquons que, de jour comme de nuit, la lumière ne sait que glisser sur les eaux: "Déjà j'imaginais ma veille silencieuse derrière les vitres, tandis que la croix des faisceaux balaierait les dunes, puis traverserait la mer" (Lods, 1991: 154). La lumière ne pénètre pas l'obscurité de l'eau, elle ne malmène que l'obscurité "au-dessus de la mer" (ibid.: 221). De l'œil dans la nuit que représentent la cabine et son occupant, à l'œil de la nuit et au veilleur du monde, il n'y a qu'un pas. Le fait de se poster dans le seul point lumineux place le sujet regardant au cœur de l'obscurité. La mer, les rayons qui émanent des lentilles du phare et le personnage constituent les seuls

\footnotetext{
${ }^{15}$ Ce thème est développé dans: Gendre, 2007: 377.

${ }^{16}$ Des exemples sont proposés dans Gendre, 2007: 278-279.
} 
éléments mobiles de ce paysage nocturne. Le verbe "traverser" implique en filigrane l'autre côté, le continent qui n'arrête pas le regard, pas plus qu'il n'arrête les flots.

Sans profondeur, la mer n'en est pas moins dépourvue de danger. Un bref rappel climatique peut apporter un élément d'explication quant à la fascination qu'exerce la surface des eaux, pour l'insulaire de la zone tropicale: associée à la charge d'humidité des nuages, cette plaine liquide est à l'origine des ouragans et des cyclones.

Dans les récits de Jean Lods, l'écran marin semble constituer une manifestation de l'incidence de l'environnement naturel sur l'appréhension inconsciente et imaginaire que le sujet a de l'espace dans lequel il évolue. En plein jour, le tableau que réalise le phare évoque la toile Lighthouse Hill ${ }^{17}$ d'Edward Hopper, en raison des frontières rectilignes qu'imposent à l'espace la verticalité de l'édifice et l'horizontalité de son ombre barrée par la mer: "Quand je remontai, le disque du soleil s'était complètement détaché du continent, l'ombre du phare s'allongeait sur le sable jusqu'à la mer, et la lumière qui baignait la cabine à travers les vitres faisait mal aux yeux" (ibid:: 186). Le contraste entre l'île et le continent est ici le fait de la trajectoire de l'astre solaire: sa séparation de la terre s'oppose à la jonction de l'ombre du bâtiment avec le rivage. La réverbération de la lumière sur la cellule de verre redouble l'éblouissement du soleil. Soleil dans la nuit, le phare apparaît tout à fait, dans cette contemplation diurne, comme un avatar de l'astre. De plus, à l'instar des terres, continentales et insulaires, ainsi que du soleil et de la mer, il appartient au paysage naturel.

La fin du vide constitue aussi ce lieu où se perdent les mots non articulés. Précieuses sont pour l'écrivain les tentatives de repousser l'indicible. Or, leurs manifestations, euphoriques ou dysphoriques, sont captées par les flots. Euphoriques, sur le paquebot, l'accompagnement au piano ainsi que les interjections heureuses que suscite la musique. Mais les sons s'évanouissent: "les 'ah!' admiratifs qui prolongent chaque note abandonnée à son sort dans la nuit de l'océan Indien" (Lods, 1987: 27). La soirée festive, ses atours de séduction et ses déploiements d'originalités individuelles, contrastent avec le géant marin. Les mondanités n'en paraissent que plus futiles; les êtres, davantage perdus au milieu de l'élément omnipotent. Le narrateur ne partage pas, ni ne se laisse gagner par l'euphorie des personnages. Tout à fait dysphorique, en revanche, ce plan en arrêt d'Emmanuel: "Debout sur la crête des dunes, immobile, son manteau secoué par le vent, il contemple fixement l'océan comme s'il en attendait une réponse" (Lods, 1991: 171). L'élément et l'Homme semblent se toiser. Le vêtement, objet inanimé et agité par la brise, constitue le seul indice qui dément l'image fixe. De plus, le contraste mouvement / immobilité dramatise le vis-à-vis.

Le narrateur vit de façon récurrente ce stationnement frontal face au limen que représente la mer, comme s'il obéissait à une force d'attraction. En témoigne l'une des

\footnotetext{
${ }^{17}$ Dans sa monographie, Rolf Günter Renner (Günter Renner, 1993) compare avec une grande finesse cette toile à La nostalgia dell' infinito, de Giorgio de Chirico.
} 
dernières phrases de Sven: "Traînant un fauteuil jusqu'à la baie, je m'installai face à la mer" (ibid:: 224). En disposant le meuble, Emmanuel adopte l'attitude du spectateur passif. La vitre se fait écran filmique sur lequel se déploie la marine cinétique. La transparence du verre tend à faire disparaître, pour l'œil, la frontière qui sépare l'intérieur et l'extérieur, tandis que sa matière protège l'observateur du contact avec la réalité du dehors. L'œil est dehors, alors que le corps est à demeure dedans, privé de tout contact avec l'objet observé. Le narrateurpersonnage se fait ici voyeur, mais voyeur de l'obscurité, c'est-à-dire de ce qui ne se laisse pas saisir par la perception visuelle.

C'est également ainsi qu'apparaît la mer, insondable et invisible, au narrateur du Bleu des vitraux, lorsqu'il se remémore sa fuite dans le cirque, à la fin de la fiction: "Plus bas commencent les champs de cannes, et au-delà, je la vois, elle est là. La mer. Comme un bandeau miroitant de soleil entre les arbres ou au-dessus des sabres rêches des cannes" (Lods, 1987: 187). A l'instar d'une île, le tableau la saisit encadrée par des lignes végétales. On remarque dans cette occurrence l'ébauche d'un motif exploré explicitement dans d'autres évocations de la mer, celui du prolongement qu'elle tend à offrir à l'île. Le corps et l'œil dehors du personnage remémoré doivent être distingués de la voix narrative dans le temps présent de l'énonciation et de l'œil de la mémoire. La mer éblouissante convoquée est aussi invisible que la mer du Nord dans la nuit. Invisible d'être trop visible, la réverbération de la mer dans l'océan Indien est aussi le fait de la richesse en phosphore de la mer Rouge. A l'image d'un éclairage au néon, elle livre crûment les êtres au regard: "Les reflets de la mer Rouge, s'évasant à partir du hublot, se reflètent sur la peinture laquée des cloisons et posent un halo indiscret sur son visage et ses bras nus" (ibid.: 24). La mise à nu ressortit également au polyptote (reflet, se reflètent): la surface de l'eau ne renvoie qu'à elle-même et s'étend sur tout, sur l'animé comme l'inanimé. Au moyen de l'adjectif "indiscret", le narrateur attribue l'impudeur à la source de lumière. Cependant, celle-ci influe sur le choix des objets regardés: l'œil s'empare des parties du corps qui sont découvertes. Ainsi les caractéristiques de l'espace environnant influencent-elles explicitement la qualité du regard.

Faut-il reconnaître dans cet invisible une fascination pour ce qui se dérobe, résiste à toute tentative d'appropriation? La mer ne se possède pas: elle ne relève pas de l'ordre de l'avoir. Elle confine l'être dans ses retranchements. L'objet du regard n'est pas tant la mer que le regard lui-même qui est posé sur ce limen. Preuve en est la référence marine qui ressortit à la fois au personnage, au narrateur et à l'auteur et qui envisage l'élément comme une instance mimétique complexe. En effet, la mer gagne les terres. Le continent occidental, que devine, grâce à ses jumelles, le vieux gardien du phare des Wadden, lui apparaît comme englouti par l'eau: "Il observa un long moment la mince ligne sableuse qui émergeait à peine de la mer" (Lods, 1991: 64-65). L'étendue de la mer est telle que le continent prend l'aspect d'une île naissante. Si l'élément marin est observé par un regard en surplomb, cette 
hiérarchie verticale de l'espace met en valeur la contamination des plaines: "De là-haut on domine la plaine jusqu'à la mer, et les vagues des cannes moutonnent sur la pente avant d'aller mourir au bord des galets de la plage que raclent les rouleaux poussés par l'océan" (Lods, 1987: 22-23). La métaphore filée ("vagues", "moutonnent") est éloquente. La flore et l'eau tendent à se confondre. En revanche, dans Quelques jours à Lyon, bien que l'on retrouve les termes de la métaphore citée ci-dessus, les ordres naturels convergent vers la mer qu'ils miment chacun à leur façon: "En contrebas du parapet qui borde la route, les vagues de palmes moutonnent jusqu'à la mer, et, au-delà, la nappe éblouissante de l'océan se perd dans le ciel" (Lods, 1994a: 27). L'écume passée sous ellipse est à l'origine de l'association, stéréotypée, des vagues à la laine des moutons, qui est ici appliquée à la végétation. La réverbération de la surface liquide est identifiée à une "nappe". Le narrateur applique à la mer l'analogie passée dans l'usage de la condensation formée par le brouillard avec l'étoffe protectrice. Le ciel contamine l'océan.

Ce phénomène progresse, dans Sven, au point que le ciel se fait le miroir de la mer: "Les nuages étaient au-dessus de la mer maintenant, la plombant d'un filet sombre aux mailles d'écume" (Lods, 1991: 84). La métaphore repose de nouveau sur une analogie formelle avec une étoffe ("filet, mailles"). L'écran de la mer-miroir se reflète dans le miroir du ciel. Sven attribue cette forme de contamination à la contiguïté ténue entre les ordres naturels. En pleine nuit, le phare joue un rôle déterminant dans cette proximité: "un phare dont les faisceaux balaient les sables d'une île avant de se perdre dans la mer..." (ibid:: 162). L'éclairage est aussi hasardeux sur les terres et sur la surface des eaux: les verbes balayer et se perdre dans en témoignent. Sans la lumière artificielle, ni la terre ni la mer, absolument confondues, ne font signe l'une de l'autre. L'aube est précisément le temps de la différenciation: "Je guettais, immobile, pendant tous ces instants où les dunes sortaient de la nuit, où le ciel se séparait de la mer, où l'écume recommençait à galonner l'île" (ibid.: 185). L'ornithologue choisit le crépuscule du matin pour scruter les paysages. Le verbe de la proposition principale, guetter, est utilisé dans son emploi absolu, intransitif. Ce "matin du monde" coïncide avec la vigilance exacerbée du personnage. Les trois propositions subordonnées temporelles réalisent un rythme ternaire à cadence majeure qui scande l'euphorie du moment. Elles correspondent aux trois étapes de la révélation d'une image en trois dimensions qui superposent successivement les reliefs, l'altitude et les couleurs. Cette phrase, incipit de la séquence 20 du récit, apporte des éléments d'explication quant au "mal de l'île".

L'indifférenciation des éléments qui composent l'espace menace en effet les repères fondamentaux de l'être qui se voit confronté à plusieurs formes d'invisibilité, synonymes d'autant de cécités: invisibilités dues aux cycles journaliers doublées d'invisibilités affectives. Sans doute le mimétisme de la mer explique-t-il la mimésis qui caractérise la convocation de 
l'élément en tant que modèle à suivre pour préserver l'intégrité du moi. Dans l'isolement du cirque, Sven construit son univers de références en observant l'environnement naturel qui l'entoure. Ses défenses psychiques puisent d'une part dans le fonds imaginaire dans lequel la nature détient un rôle dominant (la forêt, par exemple), mais calquent par ailleurs leur mise en œuvre sur les phénomènes naturels observés: "Il appelle au secours les images de la forêt qu'il rêve pour repousser les scènes étrangères, pour les dissoudre, pour les recouvrir comme la mer recouvre le sable" (ibid.: 116). Le personnage s'efforce de contraindre la mémoire involontaire ("les scènes étrangères") au moyen de souvenirs choisis. Le flux et le reflux deviennent l'emblème de cette mémoire volontaire et protectrice: la reprise du verbe "recouvrir" dans le comparé et le comparant parachève leur identité. L'élément se voit associé, ici de façon explicite et dans une comparaison, au processus mémoriel. La part destructrice de son ambivalence est de ce fait nuancée: l'effacement, action suppressive, est envisagé comme salvateur.

La singularité du limen marin réside dans l'investissement dont il est à la fois l'objet et l'enjeu dans le récit lodsien: l'ambivalence avalage / fonction génésique peut être appréhendée comme l'action, tandis que tous ces météores véhiculés par le ventre bleu en seraient les signes. L'espace insulaire les retient, et c'est grâce à eux que son existence se manifeste. Les montagnes de l'île tropicale arrêtent les nuages. Ceux-ci dominent également la mer qui entoure le morceau de terre. La captation visuelle de la mer serait difficile pour l'insulaire sans ces nuées qui la surplombent. Le regard peut choisir et s'approprier des détails qui les caractérisent. Depuis l'avion, les nuages apparaissent comme des révélateurs de l'île: "On était encore au-dessus de la mer, mais là-bas, au-delà du bord argenté de l'aile, on voyait l'île apparaître avec ses pics sombres crevant une couronne de nuages laiteux" (Lods, 1987: 47). Le contraste est parachevé par l'antithèse des adjectifs "sombres" et "laiteux", simultanée à celle de la verticalité des sommets et de l'horizontalité des nuages. Mais ce tableau trouve un écho dans les circonvolutions des oiseaux sous l'œil avide de l'ornithologue: "Dans le ciel au-dessus de la mer des Wadden, des goélands tournaient sans fin, tranchant l'air du couteau de leurs ailes" (Lods, 1991: 17). Notons que les oiseaux désertent en général la forêt lodsienne. Ceux-ci préfèrent à ses moiteurs humides les hauts horizons sur la surface liquide. La métaphore qui identifie l'aile à une arme blanche participe de la netteté de la vision. Contrairement à la mer, l'air peut se fendre. S'il constitue un double de la mer, à la différence de celle-ci, il ne suscite aucune inquiétude. Cette représentation du ciel nordique, dans Sven, est tout à fait éloquente: "Il lâche la sterne dans sa pensée et la suit jusqu'à ce qu'elle ait disparu à grands coups d'ailes rapides derrière le large drap bleu de la mer tendu au fil de l'horizon" (ibid:: 110). Le caractère imaginaire de la délivrance de l'oiseau est dénoncé explicitement par l'expression "dans sa pensée". 
Pour le rêveur, l'envol s'achève quand le regard rencontre l'obstacle de la mer. Magiquement, celle-ci arrête en même temps l'objet vu et l'instance voyante. La présence du volatile parachève la métamorphose de la mer en espace de signes. Déplions encore un peu ce qui se joue là, sans cesse et incidemment, à fleur de texte. Si l'ornithologie semble constituer un alibi de l'attente d'Emmanuel, les oiseaux sont ses seuls compagnons dans cette lande d'isolement. Les espèces observées sont énumérées ici en fonction de leur lieu de nidation, de sorte que cette escale insulaire nordique s'impose comme une aire de gestation. Emmanuel recherche en elles ce qui lui est familier. Seuls l'intéressent les sites qu'elles choisissent pour procréer. Tandis que leur chant reste silencieux, l'attente se transforme en surveillance de leur gestation. Les hommes mûrs de Sven illustrent chacun une forme de maladie de la paternité. Pour Emmanuel, celle-ci se manifeste notamment dans son observation des oiseaux: il contemple en eux des cellules familiales solidaires. De nids en nids, il est sensible à la cohésion des oiseaux et au fait qu'ils partent tous ensemble. Dans cette espèce animale, il n'est pas de laissé pour compte: "La douzaine de petites oies des moissons que je surveillais depuis leur arrivée, deux jours plus tôt, n'allaient sans doute pas tarder à reprendre leur route vers la Scandinavie" (ibid.: 68). L'île n'est qu'une escale provisoire.

II n'est pas anodin que la dernière séquence du récit s'ouvre sur l'évocation du regard d'Emmanuel qui pense discerner en deux formes blanches des "cygnes de Bewick" (ibid.: 215). II s'agit du "premier couple de la troupe". La primauté associée à la symbolique universelle du cygne qui fait de cet animal le représentant de "la lumière mâle, solaire et fécondatrice" (Chevalier et Gheerbrant, 1993: 332) corrobore la fonction attribuée aux oiseaux qui dépasse la simple couleur locale. Ils portent et étayent à la fois le thème principal qu'explore le récit: la paternité. Or, c'est précisément leur jeu avec les éléments qui reflète leur pouvoir. II est tel qu'une métaphore filée attribue les caractéristiques de la mer aux airs dans lesquels ils évoluent, si bien que: "Deux goélands [...] s'envolèrent à mon approche, se laissèrent soulever par un courant, et, inclinant de l'aile, dérivèrent vers l'intérieur de l'île" (ibid:: 216). Les frontières qui distinguent les différents composants de l'espace tendent à s'effacer. Le havre insulaire ressortit à la possibilité de cette dérive sereine, qu'il offre aux oiseaux, mais aussi aux êtres humains qui l'habitent. La dernière page du récit convoque la réitération vespérale de ce spectacle: "Devant moi, comme tous les soirs, l'air était rempli des allées et venues d'oiseaux au-dessus de Mokbaaï' (ibid.: 224). II semble tirer son importance de figurer dans la narration cette singulière dérive qui préside à l'acte d'écriture. C'est pourquoi il est juste d'envisager la textualisation du miroir céleste maritime et de la mer de sable septentrionale, comme une image dupliquée de la textualisation de l'acte d'écriture au sein du récit. 
Les mimétismes marins révèlent la complexité de la référentialité interne au tissu textuel, d'une part en raison de la polyphonie qui les produit et qui orchestre la partition marine, d'autre part parce qu'ils interrogent le référent là où précisément il vient à manquer. La surface du limen offre un écran paradoxale, dans la mesure où justement son opacité donne à voir, tandis que son pouvoir aveuglant est précisément ce qui seul est visible. Miroir marin, il reflète le miroir du ciel. Ainsi cette mer imaginaire livre-t-elle à l'infini une fin du vide. Favorisée par la contigüité des éléments, cette fin du vide "au bout de l'île" inaugure la mise à l'épreuve de l'acte d'écriture dans l'image-même de l'espace-signe que réalise le limen marin. Se cristallise en lui toute l'aventure de l'écriture, sans rien oublier de ses effacements.

\section{Conclusion}

La partition de l'espace dans laquelle s'inscrit la représentation de l'élément marin dans les récits de Jean Lods montre combien ses nombreuses convocations répondent à des enjeux narratifs qui ne sauraient le réduire à une simple toile de fond. La marine lodsienne rompt également avec la stéréotypie édénique que lui ont longtemps associée les littératures exotique et coloniale. Si la mer participe ici des événements et de la construction des personnages, elle accompagne en s'y substituant parfois les mouvements de la conscience des personnages comme des narrateurs. L'observation de l'ambivalence avalage / fonction génésique précise ses affinités avec l'être-étant en mettant en relief d'une part le temps primordial réintroduit dans le texte à la faveur de la plupart de ses manifestations, et parallèlement, sa puissance à générer les possibles, qu'ils soient créatifs (dans le motif de la naissance de l'espace insulaire) ou destructeurs (la tempête, l'érosion sans fin), de sorte qu'elle devient emblématique du pouvoir générateur. La complexité de la référentialité textuelle qui affecte ce ventre-bouche l'impose comme le grand orchestrateur de la fin du vide. L'image de l'aventure de l'écriture en cours traverse les textes de Jean Lods précisément grâce à l'exploration originale de cette caractéristique prêtée au limen marin. Ces trois étapes de notre réflexion déplient les liens intimes qui unissent la représentation textuelle de l'élément marin et l'une des manifestations de la textualisation de l'acte d'écriture.

De même qu'il ne se contente pas de convoquer l'île de La Réunion des années 1940-1950, l'univers lodsien dépasse une opposition binaire de l'océan Indien et de la mer du Nord. Sven apporte en effet cet éclairage majeur: l'épiphanie de l'île du Nord est le fait de l'enfance isolée dans le cirque tropical: c'est cette répartition de l'espace construite avec la solitude qui envisage, qui impose son déchiffrage du paysage et le recrée. Dans la convocation d'un espace, s'invite toujours, à l'instar d'un filigrane, la représentation d'un autre espace. Une dialectique nouvelle se dessine ainsi et travaille autant le texte qu'elle 
soumet certaines des épreuves que rencontre l'acte d'écriture. A la différence de la littérature exotique coloniale, l'écriture de Jean Lods ne se livre pas à une description d'un espace inconnu au moyen de rapprochements plus ou moins convaincants avec un espace prétendument connu. D'une fiction à l'autre, l'originalité féconde de l'appréhension subjective de la mer réside dans le fait que l'auteur rompt tout à fait avec l'identification romantique du sujet à l'élément marin ou aux composants qui lui sont attribués. D'une facture symboliste, la marine lodsienne se fait espace de signes et espace-texte. A la fois expressionniste et impressionniste, dès qu'elle révèle et participe de l'intériorité des personnages et que paradoxalement, elle dénonce pudiquement la présence de l'aventure de l'acte d'écriture, au cœur même du texte qu'il produit.

Dans quelle mesure cette représentation de l'élément marin est-elle datée historiquement? La mer partage avec l'ensemble de la géographie imaginaire plusieurs ambivalences, comme celle du dedans/dehors. Elle invite à prendre en compte des paramètres nouveaux pour envisager l'extérialité qui caractérise la géographie fictionnelle lodsienne. Certes, le littoral tropical, marqué par l'économie coloniale, se distingue du fondu que réalisent la mer de dunes et la mer septentrionale. Mais dans cette géographie fictionnelle, la lisière de l'île du nord est signe d'un espace vierge, anhistorique et hors mémoire. Si l'espace nordique rappelle que l'origine est toujours seconde, la représentation de la mer inscrit là la géographie imaginaire dans la problématique du temps primordial: l'île septentrionale est-elle promesse d'un dépassement de l'origine ou promesse du retour de l'originel? 


\section{Bibliographie}

BLANCHOT, Maurice (1998). L'Espace littéraire. Paris: Gallimard (Folio essais).

CELINE, Louis Ferdinand (1989). Voyage au bout de la nuit. Paris: Gallimard (Folio).

CheVAlier, Jean et GheERBRANDT, Alain (1993). Dictionnaire des symboles, mythes, rêves, coutumes, gestes, formes, figures, couleurs, nombres. Paris: Robert Laffont (Bouquins).

DURAND, Gilbert (1990). Les Structures anthropologiques de l'imaginaire. Introduction à l'archétypologie générale. Paris: Dunod.

EVE, Prosper (2003). Les Esclaves de Bourbon, La mer et la montagne. Paris: Karthala et Réunion: Université de la Réunion (Collection Tropiques).

GENDRE, Annick (2007). “L'Espace d'une vie, biographie-fiction de Jean Lods” [en ligne]. In: La Part de Lods [consulté le 29/12/2008] <URL: http://dunerivelautre.free.fr/index_fichiers/page10.htm>.

GENDRE, Annick (2007). La Représentation de soi à travers la textualisation de l'espace insulaire réunionnais: étude de l'œuvre de Jean Lods. Thèse de doctorat [en ligne]. In: TEL [consulté le 17/12/2008] <URL: http://tel.archives-ouvertes.fr/ index.php?halsid=56k1 cirvvrtr10qsrofcqtb0l7\&view_this_doc=tel-00174119\&version $=1>$.

GÜNTER RENNER, Rolf (1993). Edward Hopper 1882-1967. Métamorphoses du réel. Cologne: Benedikt Taschen Verlag.

LODS, Jean (1973). Le Silence des autres. Paris: La Pensée universelle.

LoDS, Jean (1977). La Part de l'eau. Paris: Gallimard.

LODS, Jean (1980). La Morte saison. Paris: Gallimard.

LODS, Jean (1987). Le Bleu des vitraux. Paris: Gallimard.

LODS, Jean (1990). "L'Espoir transculturel: Ecriture et déracinement", prononcée à Saint Gilles les Bains, 7 juillet 1988. In: L'Espoir transculturel, cultures, exils et folies dans l'Océan Indien, Tome 1: Santé mentale, représentation de la maladie et itinéraires thérapeutiques, sous la direction de Jean-François Reverzy. Paris: coédition INSERM, ARRPPPOI, L'Harmattan.

LODS, Jean (1991). Sven. Paris: Calmann-Lévy.

LODS, Jean (1992). "Clair de lune”. In: Chants pour une île qui n'existe pas. Saint-Denis (La Réunion): Editions UDIR.

LODS, Jean (1994a). Quelques jours à Lyon. Paris: Calmann-Lévy.

LODS, Jean (1994b). Mademoiselle. Vénissieux: Editions Paroles d'Aube, en coédition avec Grand Océan, Saint-Denis.

SIMON, Claude (1997a). La Route des Flandres. Paris: Editions de Minuit.

SIMON, Claude (1997b). Le Jardin des Plantes. Paris: Editions de Minuit. 